\title{
Phenotypic and Molecular Characterization of Extended-Spectrum $\beta$-Lactamase Produced by Escherichia coli, and Klebsiella pneumoniae Isolates in an Educational Hospital
}

\author{
Abolfazl Gholipour '; Neda Soleimani ${ }^{2}$; Dariush Shokri ${ }^{3}$; Sina Mobasherizadeh ${ }^{4}$; \\ Mohammad Kardi ${ }^{2}$; Azar Baradaran ${ }^{2, *}$ \\ ${ }_{2}^{1}$ Department of Microbiology and Immunology, Cellular and Molecular Research center, Faculty of Medicine, Shahrekord University of Medical Sciences, Shahrekord, IR Iran \\ ${ }_{3}^{2}$ Department of Pathology, Al-Zahra Hospital, Isfahan University of Medical Sciences, Isfahan, IR Iran \\ ${ }^{3}$ Nosocomial Infection Research Center, Isfahan University of Medical Sciences, Isfahan, IR Iran \\ ${ }^{4}$ Department of Microbiology, Al-Zahra Hospital, Isfahan University of Medical Sciences, Isfahan, IR Iran \\ ${ }^{*}$ Corresponding author:Azar Baradaran, Department of Pathology, Al-Zahra Hospital, Isfahan University of Medical Sciences, Isfahan, IR Iran. Tel: +98-3116691565, Fax:+98-3116684510, \\ E-mail: azarbaradaran@yahoo.com
}

Received: July 16, 2013; Revised: September 042014 ; Accepted: September 08, 2014

Background: Extended-spectrum beta-lactamases (ESBLs) are a group of enzymes that hydrolyze antibiotics, including those containing new cephalosporins, and they are found in a significant percentage of Escherichia coli and Klebsiella pneumoniae strains. With the widespread use of antibiotics, difficulties with infection therapy caused by drug resistant organisms, especially those that have acquired resistance to beta-lactams, such as broad-spectrum cephalosporins, have amplified the above-mentioned organisms.

Objectives:This study was conducted to characterize ESBLs among E. coli and K.pneumonia isolates by molecular and phenotypic methods. Materials and Methods: Different strains of E. coli and K. pneumonia were collected from patients with urinary tract infections. The ESBL phenotype was determined by a double disk diffusion test (DDDT). In addition, polymerase chain reaction (PCR) analysis specific for $\beta$-lactamase genes of the TEM and SHV family was carried out. The PCR products were run on agarose and examined for DNA bands.

Results: A total of $245 \mathrm{E}$. coli and $55 \mathrm{~K}$. pneumonia strains were isolated from different samples. In total, 128 of the 300 isolates were confirmed as potential ESBLs producers as follows: 107 (43.67\%) E. coli and 21 (38.18\%) K. pneumonia. ESBLs genes were found in 24 isolates (18.75\%): 21 E. coli and 3 K. pneumonia isolates. The TEM gene was present in $13(12.14 \%)$ E. coli strains, but it was not detected in K.pneumonia. In addition, the SHV gene was present in $8(7.47 \%)$ E. coli and $3(14.28 \%)$ K. pneumonia isolates. Five (4.67\%) of the E. coli isolates harbored both TEM and SHV genes. All isolates (100\%) were susceptible to imipenem. The lowest rates of resistance to other antibiotics were observed for; piperacillin-tazobactam (6.25\%), amikacin (12.5\%) and gentamicin (14.84\%). The rates of resistance to other antibiotics were as follow: nitrofurantoin (16.4\%), nalidixic acid (23.43), co-trimoxazole (25\%), cefepime (32\%), ciprofloxacin (55.46\%), ampicillin (69.53\%), ceftazidime (100\%), and cefotaxime (100\%).

Conclusions: The results of this study indicate the widespread prevalence of ESBLs and multiple antibiotic resistance in E. coli and K. pneumoniae. Therefore, beta-lactam antibiotics and beta-lactamase inhibitors or carbapenems should be prescribed based on an antibacterial susceptibility test.

Keywords: Phenotypic; Molecular; extended-spectrum $\beta$-lactamase (ESBL); Escherichia coli; Klebsiella pneumoniae

\section{Background}

The difficulties seen in combating infections caused by drug resistant organisms, especially those that have acquired resistance to beta-lactams, such as broad-spectrum cephalosporins, have resulted from the extensive use of broad-spectrum antibacterial agents. One important resistance mechanism to the beta-lactams, including new cephalosporins, is due to the destruction of the antibiotics by extended-spectrum beta-lactamases (ESBLs) (1). ESBLs were first described in the early 1980 s among Klebsiella species, and then in Escherichia coli, Serratia marcescens, Pseudomonas aeruginosa, and other Gram-negative bacilli $(2,3)$. ESBLs are a group of enzymes that have an expanded substrate profile which allows for the hydrolysis of 3 and 4 generation cephalosporins and monobactams, but not carbapenems. ESBLs are prevented by $\beta$-lactamase inhibitors, such as; clavulanic acid, sulbactam and tazobactam $(2,4)$. These enzymes can be either plasmid or chromosomally mediated, but they are described mainly on plasmid that are frequently found among Enterobacteriaceae.

ESBLs are prevalent in every part of the world, and in addition, they are found in a meaningful percentage of E. coli and Klebsiella pneumoniae strains in certain countries $(4,5)$. ESBLs are continuous mutations that change the amino acid configuration near the active site of these $\beta$-lactamases, resulting in the development of new enzymes showing extended substrate profiles. Until now, more than 400 different ESBLs have been identified, and

Copyright @ 2014,Ahvaz Jundishapur University of Medical Sciences; Published by Kowsar Corp. This is an open-access article distributed under the terms of the Creative Commons Attribution License, which permits unrestricted use, distribution, and reproduction in any medium, provided the original work is properly cited. 
these are clustered into three groups: TEM, SHV and CTXM, with 183, 134 and 103 variants, respectively. Among the previously mentioned ESBL variants, TEM and sulphydryl variable SHV were the major types in some countries (6-8).

Determination of ESBL genes, including TEM and SHV, by molecular techniques in bacteria that produce ESBL and their antimicrobial resistance patterns can provide applicable information about their epidemiology and risk factors related to their infections $(2,9)$. A number of studies have been carried out to recognize the types of ESBL producing Enterobacteriaceae in hospitals in Iran $(1,10,11)$. In spite of the presence of ESBLs among K. pneumoniae and E. coli which have been reported from Isfahan (12), there is no information on their molecular types.

\section{Objectives}

The present study was conducted to determine the prevalence of TEM and SHV genes responsible for ESBL production amongst ESBL positive K. pneumoniae and E. coli isolated from urinary tract infection specimens of both hospitalized patients and outpatients.

\section{Materials and Methods}

\subsection{Bacterial Isolates}

In this study, E. coli and K. pneumonia strains isolated from patients suffering from urinary tract infections were studied. The isolates were collected from December 2011 to October 2012 from Al-Zahra Hospital, Isfahan. Tests were conducted on both hospitalized and non-hospitalized infections. Hospitalized infections were defined as patients who were confined to bed in hospital, while non-hospitalized infections were defined as infections in patients who had had no previous contact with hospitals or long-term care facilities in the previous two weeks. Bacterial isolates were characterized using biochemical tests. The samples were cultured on nutrient agar (Hi Media, India), MacConkey agar (Hi Media, India), blood agar (Hi Media, India) and eosin methylene blue (EMB) agar (Hi Media, India). The plates were incubated at $35^{\circ} \mathrm{C}$ for $24 \mathrm{~h}$ and the pure isolates characterized and identified according to Gram stains and biochemical tests such as; catalase, oxidative, indole production, citrate utilization, triple iron sugar, urea test, oxidative-fermentative test with glucose, orthonitrophenyl- $\beta$-galactoside (ONPG) test, and methyl red Voges-Proskauer, as described in standard bacteriological methods. All of the above chemicals and media were purchased from Sigma-Aldrich (Germany).

\subsection{Antimicrobial Drug Susceptibility Testing}

Antimicrobial susceptibility of the isolated bacteria was performed by the disk diffusion technique on MuellerHinton agar plates (Oxoid, UK), according to the recommendations of the Clinical and Laboratory Standards Institute (CLSI). The antibiotics ( $\mu \mathrm{g})$ tested included: ami- kacin (30), ampicillin (10), ciprofloxacin (5), co-trimoxazole (10), gentamicin (10), imipenem (10), nitrofurantoin (300), tazocin (110), ceftazidime (30), cefepime (30), nalidixic acid (30), and cefotaxime (30). The standard antibiotic disks were purchased from Mast Diagnostics (Mast Group, UK).

\subsection{Phenotypic Screening of Extended-Spectrum $\beta$-lactamase}

The isolates that showing resistance to one or more third generation cephalosporins (3GCs) were examined for ESBLs production by the combination disc method using; cefotaxime (30), cefotaxime/clavulanic acid (30/10), ceftazidime (30), and ceftazidime/clavulanic acid (30/10) (MAST Co. UK). A greater than or equal to $5 \mathrm{~mm}$ increase in diameter of the inhibition zone of the cephalosporinplus-clavulanate disc, when compared to the cephalosporin only disc, was interpreted as phenotypic evidence of ESBLs production. K. pneumoniae ATCC 700603 was used as a positive control and E. coli ATCC 25922 was used as a negative control. Standard strains were obtained from the American Type Culture Collection (Manassas VA).

\subsection{DNA Extraction for Polymerase Chain Reaction}

DNA templates for polymerase chain reaction (PCR) were obtained from the overnight growth of bacterial isolates on Luria-Bertani agar (Hi Media, India) that were pelleted by centrifugation, resuspended in $500 \mu \mathrm{L}$ of sterile deionized water, and then boiled for $10 \mathrm{~min}$. After centrifugation of the boiled samples at $19000 \mathrm{~g}$ for $5 \mathrm{~min}$, the upper layer was used as a DNA template for PCR.

\subsection{Polymerase Chain Reaction Amplification of $\beta$-Lactamase Genes}

Bacterial isolates included in the study were screened by PCR for TEM and SHV genes. Specific primers and annealing temperature for amplifying the blaSHV and blaTEM genes by PCR are shown in Table 1. PCR was carried out in a $25 \mu \mathrm{L}$ volume containing; $2.5 \mu \mathrm{L}$ of $10 \mathrm{X}$ PCR reaction buffer, with $\mathrm{MgCl}_{2}(1.5 \mathrm{mM}), 0.5 \mu \mathrm{L}(200 \mu \mathrm{M})$ deoxynucleoside triphosphate mix (dNTPs, $10 \mathrm{mM}), 0.5 \mu \mathrm{L}$ of each primer $(10 \mathrm{pm} / \mu \mathrm{L})$, and $50 \mathrm{ng}$ DNA template with $0.5 \mu \mathrm{L}(3 \mathrm{U} / \mu \mathrm{L})$ Taq DNA polymerase. PCR amplifications were performed on a Thermocycler TC-512 (Techne) and the PCR fragment was analyzed on 1\% agarose gel. A molecular marker (Fermentase SM024: 80 to $1000 \mathrm{~kb}$ ) was used to assess the PCR product size. PCR materials were purchased from Fermentase (Life Science, Germany).

\section{Results}

During a 10-month period, 245 E. coli and 55 K. pneumonia strains were isolated from patients with urinary tract infections. In total, 128 of the 300 isolates were confirmed as potential ESBL producers using ceftazidime/clavulanic acid and cefotaxime/clavulanic acid disks (Figure 1). 
Gholipour A et al.

\begin{tabular}{|c|c|c|c|c|}
\hline Primers & Temperature, ${ }^{\circ} \mathrm{C}$ & Nucleotide Sequences, 5'-3' & References & Size, bp \\
\hline SHV & 60 & & $(2)$ & 293 \\
\hline $\mathrm{F}$ & & CGCCTGTGTATTATCTCCCT & & \\
\hline $\mathrm{R}$ & & CGAGTAGTCCACCAGATCCT & & \\
\hline TEM & 60 & & (2) & 403 \\
\hline $\mathrm{F}$ & & TTTCGTGTCGCCCTTATTCC & & \\
\hline $\mathrm{R}$ & & ATCGTTGTCAGAAGTAAGTTGG & & \\
\hline
\end{tabular}

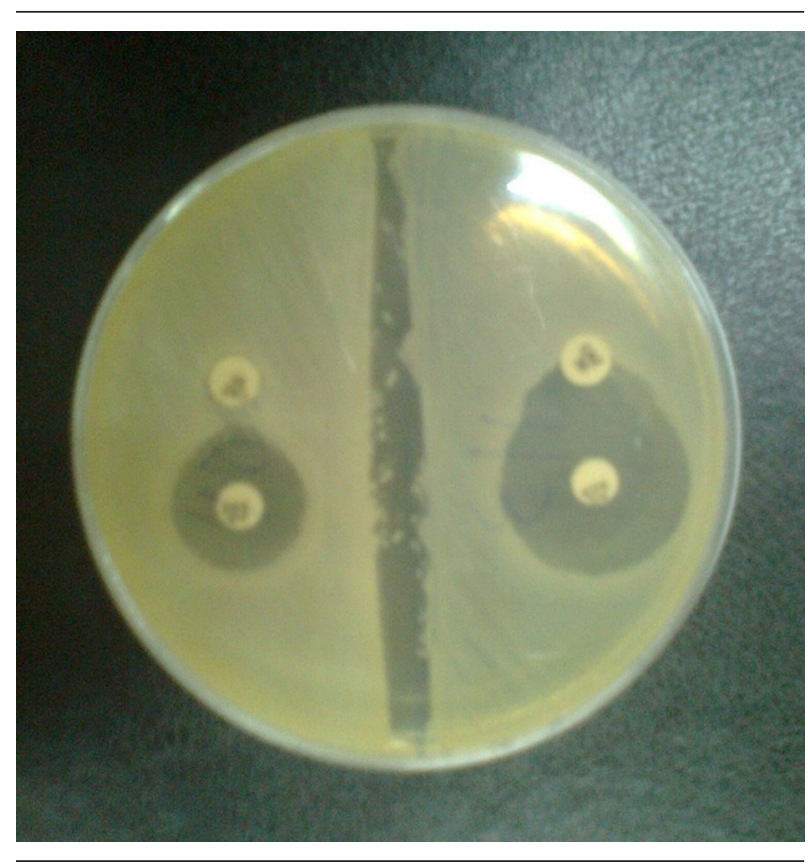

Figure1. ConfirmatoryTest for Detection of Extended-spectrum $\beta$-lactamase

The occurrence of ESBL in the isolates was as follows: 107 (43.67\%) out of $245 \mathrm{E}$. coli and $21(38.18 \%)$ out of $55 \mathrm{~K}$. pneumonia. Of the $107 \mathrm{E}$. coli, 67 (62.62\%) were isolated from hospitalized patients and the remaining 40 (37.38\%) belonged to outpatients. Furthermore, of the $21 \mathrm{~K}$. pneumonia, 13 (62\%) were from hospitalized patients, while 8 (38\%) were recovered from outpatients.

All ESBL-producing isolates were screened by PCR using blaTEM and blaSHV specific primers (Figure 2). ESBL genes were found in 24 isolates (18.75\%): 21 E. coli and 3 $K$. pneumonia isolates. The overall data revealed that the TEM gene was present in 13 (12.14\%) E. coli, but it was not detected in K. pneumonia, and the SHV gene was present in $8(7.47 \%)$ E. coli and $3(14.28 \%)$ K. pneumonia isolates. There were $5(4.67 \%)$ E. coli isolates that harbored both TEM and SHV genes. All bacterial isolates were susceptible to imipenem. The lowest rates of resistance to other antibiotics were observed for: tazocin (6.25\%), amikacin (12.5\%) and gentamicin (14.84\%). The rates of resistance to other antibiotics were as follows: nitrofurantoin (16.4\%), nalidixic acid (23.43), co-trimoxazole (25\%), cefepime (32\%), ciprofloxacin (55.46\%), ceftazidime (59.76\%), ampicillin (69.53\%) and cefotaxime (73.43\%).

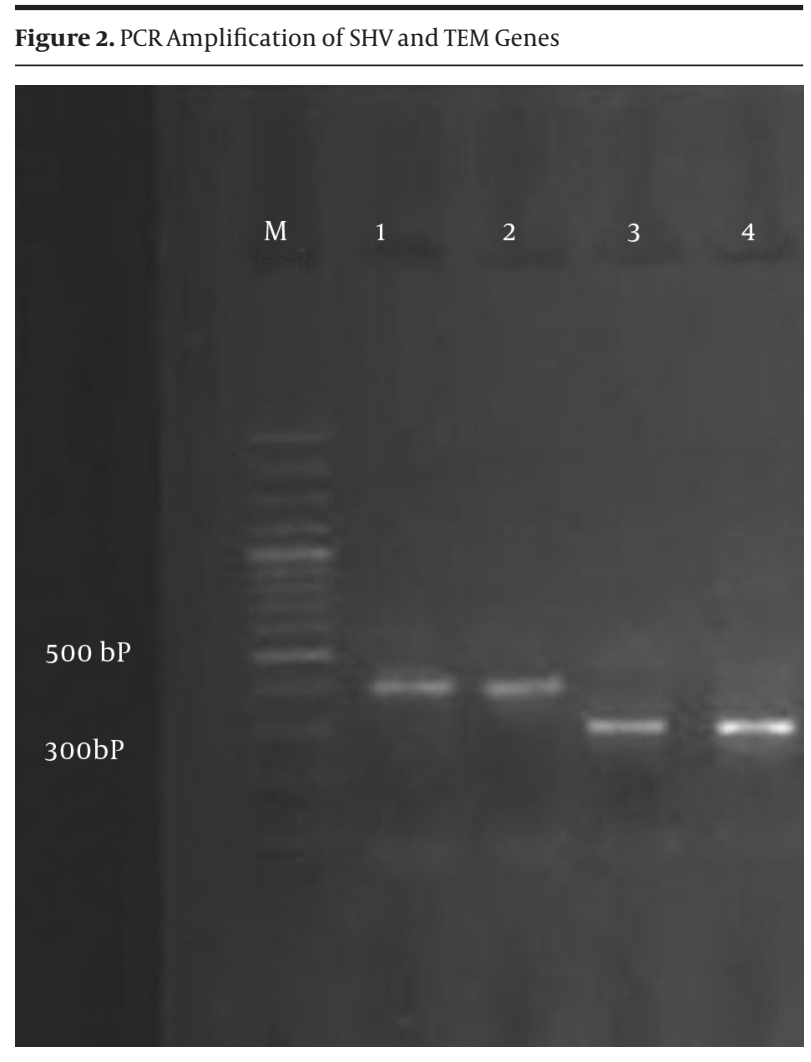

Lane M,100 bp DNA marker; Lane 1, blaSHV from the positive control; Lane 2, E. coli clinical isolates expressing blaSHV; Lane 3, blaTEM from the positive control; Lane 4, K. pneumonia clinical isolates expressing blaTEM.

\section{Discussion}

The treatment of infectious diseases is an important issue for human wellbeing and the daily increase in bacterial resistance has elevated patients' costs in recent years. The production of ESBLs is also a major threat to the use of the new generation of cephalosporins. In the last two decades, the rate of ESBL production by Enterobacteriaceae has increased considerably $(10,13,14)$. Among Enterobacteriaceae, K. pneumonia and E. coli are the most important causative agents of nosocomial infections (15) and these are usually isolated from patients with urinary tract infections in Al-Zahra Hospital, Isfahan; therefore, we selected these two types of bacteria for our study. Occurrences of infection effected by extended spectrum beta-lactamase producing K. pneumonia and E. coli have 
been widely reported all over the world following the widespread use of the expanded spectrum cephalosporins (11, 16-19).

In our study, phenotypic screening of ESBL showed that $43.67 \%$ of E. coli and $38.18 \%$ of $K$. pneumonia isolates were positive for ESBL production. Overall, $62.62 \%$ of $E$. coli were isolated from hospitalized patients and the remainder $37.38 \%$ belonged to outpatients. In addition, $62 \%$ of $K$. pneumonia were from hospitalized patients, while $38 \%$ were recovered from outpatients. Based on these results, the prevalence of ESBL producing K. pneumonia and E. coli was high. In addition, ESBLs producing organisms were higher in hospitalized patients compared to outpatients. The high prevalence of ESBLs producing E. coli and $K$. pneumonia has also been reported by a number of previous studies. For example in a study by Feizabadi et al. (1), they showed a 44.5\% ESBL positive rate among clinical K. pneumoniae isolated from clinical specimens in Tehran (20).

In a study from Kurdistan, Ramazanzadeh et al. revealed a 34.8\% ESBL positive rate among strains of Gram-negative bacteria (21) and Mobasherizadeh et al. showed that among a total of 2035 consecutive clinical isolates identified as E. coli in Al-Zahra Hospital, 898 (44.1\%) and 432 (21.2\%), were ESBL producers for hospitalized and nonhospitalized patients, respectively (22). Therefore, our findings were in agreement with the above-mentioned studies. On the other hand, the rate of ESBLs production is higher in other hospitals or cities in our country. For instance, the rates of ESBL producing $K$. pneumoniae isolated from Tehran indicated by Aminzadeh et al. were $52.5 \%$ in 2008 (23). Bazzaz et al. also showed that the prevalence of ESBL positive strains of E. coli and K. pneumonia was $59.2 \%$ in 2009 (24).

In another study that was performed by Jalalpoor and Mobasherizadeh in 2009-2010 in Isfahan (12), the frequency of ESBLs in strains of E. coli in hospitalized and out-patients was $58 \%$ and $17 \%$, respectively, and the frequency of ESBLs in strains of K. pneumoniae in hospitalized and out-patients was $64 \%$ and $22 \%$, respectively. In that study, the samples were collected from Al-Zahra, Shariati, and Kashani hospitals and reference and Mahdieh laboratories. Their study results showed a higher frequency of ESBLs in isolated bacteria from hospitalized cases when compared to out-patients. Therefore, we see that our results are compatible with the results of the previously mentioned study. The presence of ESBL positive Enterobacteriaceae was also documented by Feizabadi et al. (1). From 104 isolates of K. pneumonia collected from four university hospitals in Tehran, they identified 75 (72.1\%) ESBL producing isolates. Moreover, E. coli and K. pneumonia isolates producing ESBL are less prevalent in the Al-Zahra Hospital, Isfahan. In a study from Mashhad, Zaniani et al. revealed that $43.9 \%$ of E. coli and $56.1 \%$ of K. pneumoniae were ESBL producers and the frequency of SHV and TEM among the ESBL producing isolates were $14.4 \%$ and $20.6 \%$, respectively (25). The prevalence of ESBLs varies from one hospital to another. It is uncertain whether this is because of the differences in infection control practices between hospitals or to differences in the use of new cephalosporins (11).

Our study, along with other studies, have also demonstrated that the rates of ESBLs production in our country are different from other countries in our area, such as; India (57.1\%), Turkey (57\%) and South Korea (30\%), and published data from European countries, such as; France, Italy, the Netherlands, Germany, and Spain, as well as in the United States, Australia, Japan, Tanzania, Thailand and Pakistan, which showed a higher prevalence of ESBL-producing isolates in the present study $(22,26-28)$. Feizabadi et al. (1) found that the rates of resistance for tazocin, amikacin, ciprofloxacin, cefepime, ceftazidime, and cefotaxime were; $21.3 \%, 21.4 \%, 28 \%, 76 \%$ and $84.0 \%$, respectively. The comparison of our study results with the above-mentioned study shows that antibiotic resistance to four of the previously mentioned antibiotics is lower in our study. In addition, in another study, both non-hospitalized and hospitalized isolates were more resistant to first line drugs including; ampicillin, and trimethoprimsulfamethoxazole (22). This result, which is comparable with other studies in developing countries, is due to the widespread use of these drugs because of their low cost and easy administration.

Imipenem, amikacin and piperacillin-tazobactam were the most effective antibiotics against hospitalized ESBLproducing isolates. All ESBL-positive isolates were susceptible to imipenem, indicating that this agent is the best drug for treating serious infections caused by ESBLproducing E. coli. The carbapenem antibiotics including; ertapenem, imipenem, and meropenem, are commonly known as the first choice in the treatment of serious infections caused by ESBL-producing Enterobacteriaceae (22). The prevalence of the TEM gene in our study in E. coli was $12.14 \%$, however, this gene was not detected in $K$. pneumonia. The prevalence of the SHV gene in E. coli and K. pneumonia isolates was $7.47 \%$ and $14.28 \%$, respectively, which is lower than that reported by Karimi et al. (26\% and $15 \%$ for E. coli, and $18 \%$ and $15 \%$ for K. pneumonia, respectively) (15), 54\% and $67.4 \%$ for K. pneumonia (29) and 55.7\% and $30.7 \%$ for K. pneumonia (1). Also in this study, 5 (4.67\%) of the E. coli isolates harbored both TEM and SHV genes which is lower than that reported in India (67.3\% 2003 to 2004 ) (26), (21.8\% 2007 to 2008) in Kashan, Iran (10) and (34.7\% 2006 to 2007) in Tehran, Iran (1). Therefore, we can conclude that the production of $\beta$-lactamase can result from other types of ESBLs among isolated Enterobacteriaceae. The emergence and expansion of ESBL-producing Enterobacteriaceae including, E. coli and K. pneumoniae is an alarming issue and the use of cephalosporins against these isolates is inefficient.

Nowadays, carbapenems are the drug of choice for the treatment of serious infection diseases in Iran. However, the appearance of resistance in this group of antibiotics, especially resistance to imipenem, may restrict their pre- 
scription in the future. Another concern is the tremendous increase in the prevalence of ESBLs, as the majority of these were resistant to other antibiotics. Because of the significance of ESBL producing Enterobacteriaceae including, E. coli and K. pneumoniae and the difficulties involved with the therapy of infections caused by these bacteria, clinical laboratories should adopt the employment of a simple test on the basis of the Clinical and Laboratory Standards Institute (CLSI) suggestions for the rapid identification and confirmation of ESBL production in Enterobacteriaceae. In addition, beta-lactam antibiotics and beta-lactamase inhibitors or carbapenems should only be prescribed based on an antibacterial susceptibility test.

\section{Acknowledgements}

The authors wish to thank the staffs of Al-Zahra Medical Center, Isfahan University of Medical Sciences.

\section{Authors Contributions}

Neda Soleimani and Abolfazl Gholipour designed and performed the research. Dariush Shokri analyzed data and wrote some parts of the paper. Azar Baradaran and Sina Mobasherizadeh also provided extensive intellectual contribution. Azar Baradaran reviewed the draft too. Neda Soleimani prepared the final draft.

\section{Funding/Support}

This study was supported in part by grant 390410 from the Isfahan University of Medical Sciences.

\section{References}

1. Feizabadi MM, Mahamadi-Yeganeh S, Mirsalehian A, Mirafshar SM, Mahboobi M, Nili F, et al. Genetic characterization of ESBL producing strains of Klebsiella pneumoniae from Tehran hospitals. JInfect Dev Ctries. 2010;4(10):609-15.

2. Bali EB, Acık, L. , Sultan, N. . Phenotypic and molecular characterization of SHV, TEM, CTX-M and extended-spectrum B-lactamase produced by Escherichia coli, Acinobacter baumannii and Klebsiella isolates in a Turkish hospital. Afr J Microbiol Res. 2010;4(8):650-4.

3. Kiratisin P, Apisarnthanarak A, Laesripa C, Saifon P. Molecular characterization and epidemiology of extended-spectrum-betalactamase-producing Escherichia coli and Klebsiella pneumoniae isolates causing health care-associated infection in Thailand, where the CTX-M family is endemic. Antimicrob Agents Chemother. 2008;52(8):2818-24.

4. Bradford PA. Extended-spectrum beta-lactamases in the 21st century: characterization, epidemiology, and detection of this important resistance threat. Clin Microbiol Rev. 2001;14(4):933-51.

5. Helfand MS, Bonomo RA. $\beta$-Lactamases: a survey of protein diversity. Curr Drug Targets Infect Disord. 2003;3(1):9-23.

6. Barguigua A, El Otmani F, Talmi M, Bourjilat F, Haouzane F, Zerouali K, et al. Characterization of extended-spectrum beta-lactamase-producing Escherichia coli and Klebsiella pneumoniae isolates from the community in Morocco.JMed Microbiol. 2011;60(Pt 9):1344-52.

7. McGowan JE, Jr, Hill HA, Volkova NV, Lawton RM, Haber MJ, Tenover FC, et al. Does antimicrobial resistance cluster in individual hospitals? J Infect Dis. 2002;186(9):1362-5.

8. Paterson DL, Hujer KM, Hujer AM, Yeiser B, Bonomo MD, Rice LB, et al. Extended-spectrum beta-lactamases in Klebsiella pneu- moniae bloodstream isolates from seven countries: dominance and widespread prevalence of SHV- and CTX-M-type beta-lactamases. Antimicrob Agents Chemother. 2003;47(11):3554-60.

9. Jain A, Mondal R. TEM \& SHV genes in extended spectrum betalactamase producing Klebsiella species beta their antimicrobial resistance pattern. Indian J Med Res. 2008;128(6):759-64.

10. Khorshidi A, Rohani M, Moniri R. The prevalence and molecular characterization of extended-spectrum $\beta$-lactamases-producing Klebsiella pneumoniae isolates recovered from Kashan hospital university, Iran. Jundishapur J Microbiol. 2012;4(4):289-94.

11. Nasehi L, Shahcheraghi F, Sadat Nikbin V, Nematzadeh SH. PER, CTX-M, TEM and SHV Beta-lactamases in clinical isolates of Klebsiella pneumoniae isolated from Tehran, Iran. Iran J Basic Med Sci. 2010;13(3):111-8.

12. Jalalpoor S, Mobasherizadeh S. Frequency of ESBLs in Escherichia coli and Klebsiella pneumonia strains isolated from hospitalized and out-patients with urinary tract infection in selective centers in Esfahan (2009-2010). Razi J Med Sci. 2011;18(85):7-18.

13. Mendes C, Kiffer C, Segura A, Ribeiro J, Turner P. Klebsiella pneumoniae with multiple antimicrobial resistance. Braz J Infect Dis. 2004;8(1):109-11.

14. Putman $M$, van Veen HW, Konings WN. Molecular properties of bacterial multidrug transporters. Microbiol Mol Biol Rev. 2000;64(4):672-93.

15. Karimi A, Rahbar M, Fallah F, Navidinia M, Malekan M. Detection of integron elements and gene groups encoding ESBLs and their prevalence in Escherichia coli and Klebsiella isolated from urine samples by PCR method. Afr J Microbiol Res. 2012;6(8):1806-9.

16. Branger C, Lesimple AL, Bruneau B, Berry P, Lambert-Zechovsky $\mathrm{N}$. Long-term investigation of the clonal dissemination of Klebsiella pneumoniae isolates producing extended-spectrum beta-lactamases in a university hospital. J Med Microbiol. 1998; 47(3):201-9.

17. Niumsup PR, Tansawai U, Boonkerd N, Polwichai P, Dejsirilert S. Dissemination of extended-spectrum beta-lactamase-producing Klebsiella pneumoniae and Escherichia coli in Thai hospitals. $J$ Infect Chemother. 2008;14(6):404-8.

18. Pathak A, Marothi Y, Kekre V, Mahadik K, Macaden R, Lundborg CS. High prevalence of extended-spectrum beta-lactamase-producing pathogens: results of a surveillance study in two hospitals in Ujjain, India. Infect Drug Resist. 2012;5:65-73.

19. Wang XR, Chen JC, Kang Y, Jiang N, An SC, Gao ZC. Prevalence and characterization of plasmid-mediated blaESBL with their genetic environment in Escherichia coli and Klebsiella pneumoniae in patients with pneumonia. Chin MedJ (Engl). 2012;125(5):894-900.

20. Feizabadi MM, Etemadi G, Yadegarinia D, Rahmati M, Shabanpoor S, Bokaei S. Antibiotic-resistance patterns and frequency of extended-spectrum beta-lactamase-producing isolates of Klebsiella pneumoniae in Tehran. Med Sci Monit. 2006;12(11):BR362-5.

21. Ramazanzadeh R, Chitsaz M, Bahmani N. Prevalence and antimicrobial susceptibility of extended-spectrum beta-lactamaseproducing bacteria in intensive care units of Sanandaj general hospitals (Kurdistan, Iran). Chemotherapy. 2009;55(4):287-92.

22. Mobasherizadeh S, Shokri D, Zargarzadeh AH, Jalalpour S, Ebneshahidi SA, Sajadi M. Antimicrobial resistance surveillance among hospitalized and non-hospitalized extend-spectrum beta-lactamase producing Escherichia coli from four tertiarycare hospitals in Isfahan, Iran; 2008-2011. Afr J Microbiol Res. 2012; 6(5):953-9.

23. Aminzadeh Z, Sadat Kashi M, Sha'bani M. Bacteriuria by extended-spectrum Beta-lactamase-producing Escherichia coli and Klebsiella pneumoniae: isolates in a governmental hospital in South of Tehran, Iran. Iran J Kidney Dis. 2008;2(4):197-200.

24. Bazzaz BS, Naderinasab M, Mohamadpoor AH, Farshadzadeh Z, Ahmadi S, Yousefi F. The prevalence of extended-spectrum betalactamase-producing Escherichia coli and Klebsiella pneumoniae among clinical isolates from a general hospital in Iran. Acta Microbiol Immunol Hung. 2009;56(1):89-99.

25. Zaniani FR, Meshkat Z, Naderi Nasab M, Khaje-Karamadini M, Ghazvini K, Rezaee A, et al. The Prevalence of TEM and SHV Genes among Extended-Spectrum Beta-Lactamases Producing Escherichia Coli and Klebsiella Pneumoniae. Iran J Basic Med Sci. 2012;15(1):654-60. 


\section{Gholipour A et al.}

26. Lal P, Kapil A, Das BK, Sood S. Occurrence of TEM \& SHV gene in extended spectrum beta-lactamases (ESBLs) producing Klebsiella sp. isolated from a tertiary care hospital. Indian J Med Res. 2007;125(2):173-8.

27. Tasli H, Bahar IH. Molecular characterization of TEM- and SHVderived extended-spectrum beta-lactamases in hospital-based Enterobacteriaceae in Turkey.Jpn J Infect Dis. 2005;58(3):162-7.

28. Jeong SH, Bae IK, Lee JH, Sohn SG, Kang GH, Jeon GJ, et al. Mo- lecular characterization of extended-spectrum beta-lactamases produced by clinical isolates of Klebsiella pneumoniae and Escherichia coli from a Korean nationwide survey. J Clin Microbiol. 2004;42(7):2902-6.

29. Feizabadi MM, Delfani S, Raji N, Majnooni A, Aligholi M, Shahcheraghi F, et al. Distribution of bla(TEM), bla(SHV), bla(CTX-M) genes among clinical isolates of Klebsiella pneumoniae at Labbafinejad Hospital, Tehran, Iran. Microb Drug Resist. 2010;16(1):49-53. 